\title{
Lightning in Saturn's atmosphere
}

\author{
WU HanBo \& CHEN ChuXin* \\ CAS Key Laboratory of Geospace Environment, Department of Geophysics and Planetary Sciences, School of Earth and Space Sciences, \\ University of Science and Technology of China, Hefei 230026, China
}

Received October 19, 2012; accepted January 17, 2013

\begin{abstract}
There exists large-scale lightning in Saturn's water cloud. Based on the powerful moist convection in the water cloud, we establish a thermal-induced convective model to interpret the mechanism for the processes of charge generation and separation. We also estimate the breakdown field of Saturn's atmosphere quantitatively and interpret the discharge process.
\end{abstract}

Saturn, lightning, atmosphere, convection

Citation: Wu H B, Chen C X. Lightning in Saturn's atmosphere. Chin Sci Bull, 2013, 58: 1650-1654, doi: 10.1007/s11434-013-5808-0

Recently, there are several works which have done good study on thunderstorm [1] and its solar equivalent [2,3]. There are also a couple of researches which give a deep description of solar-terrestrial interactions [4,5]. The present study is a further exploration on deep solar space.

Existence of lightning on Saturn had been discussed since Voyager times as an explanation for the radio emissions called Saturn Electrostatic Discharge, or SEDs. Cassini data showed that SED activity is correlated with convective-looking clouds on the side of Saturn that faces the spacecraft [6,7]. Saturn's weather at high pressure levels is most likely to be driven by an internal source of energy, which exhibits thermal gradient that initiates the upward convection of water cloud [8].

The cloud structure in Saturn's hydrogen and helium atmosphere can be divided into three different cloud layers. The outermost cloud layer consists of ammonia ice particles. The lightning originates from roughly 125 to $250 \mathrm{~km}$ below the cloud tops [9]. The moist convection could be very energetic reaching vertical velocity of an order of $100 \mathrm{~m} / \mathrm{s}$ due to the internal thermal heat [8].

We assume that during the process of moist convection, the collision of ice particles would cause the charge separation; the negative or positive charges would attach to the particles of different size. As a consequence, the thermal-

\footnotetext{
*Corresponding author (email: chuxin@ustc.edu.cn)
}

induced vertical convection would cause the charges' generation and separation.

\section{Processes of charge generation and separation}

First, we assume that there would be an initial electric-field $E_{0}$ (this electric-field could be produced by trifle surplus charge due to the discharge process of last time or by other mechanism such as the ionization by radiation of radioactive elements).

Supposing that $\mathrm{H}_{2} \mathrm{O}$ is a polar molecule, let us consider a model of a spherical ice particle which is polarized by this initial electric-field. The lightning exists at a depth around $200 \mathrm{~km}$ below the cloud tops. The temperature there is ranged from 255 to $275 \mathrm{~K}$, where the water from the lower level begins to condense and freeze into the small ice particles. And during this moist vertical convection process, the collisions of the different size ice particles would cause the charge transference. Considering a model described in Figure 1 , the larger spherical particle 2 is made of ice crystal with a mass $M$. It can float in the middle and upper level of the water cloud. Particle 1 is the smaller particle driven by the upward-convection with a mass $m$.

Because particles 1 and 2 have different sizes, comparative velocity of particle 1 to particle 2 is not isotropic. The 


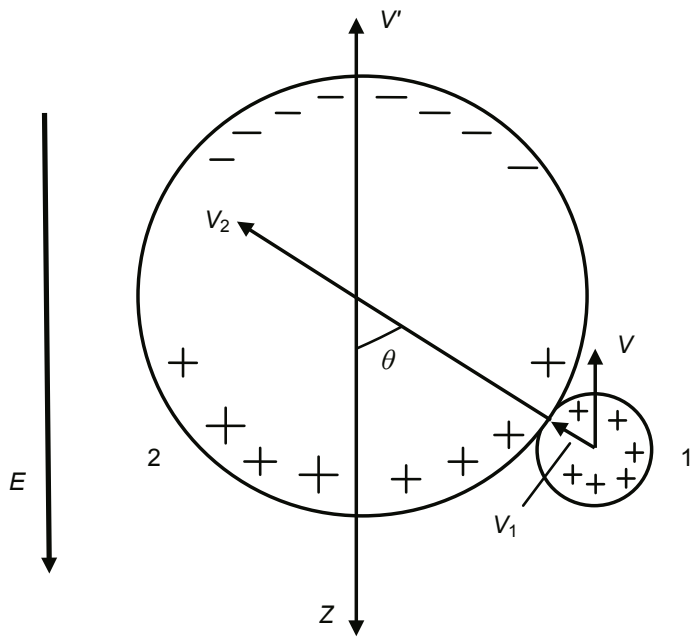

Figure 1 The angle $\theta$ is the collision angle. The exterior electric-field $E$ heads downward vertically. Particle 1 is the smaller particle driven by the powerful upward-convection with an initial velocity of $V$; particle 2 is a larger particle floating in a certain cloud level. The velocity of particle 1 after collision in the conference frame of the center of two particles is defined as $V_{1}$. The velocity of particle 2 after collision in the conference frame of the center of two particles is defined as $V_{2}$.

comparative velocity of particle 1 is directed upward. When particle 2 is polarized by outer electric-field, it has positive charge downside, and negative charge upside. Therefore particle 1 gets positive charge after the collision. If particles 1 and 2 are of the same size, then comparative velocity of particle 1 and 2 is isotropic, no charge transform will take place.

As soon as the moist convection from the bottom of the water cloud reaches a level around $200 \mathrm{~km}$ from the cloud tops, the moist air would begin to condense into the small droplets and freeze into small ice particles. These ice crystals would be brought up by the upward convection with the velocity of an order of $100 \mathrm{~m} / \mathrm{s}$. When this upward convection current meets the floating larger ice crystals, there will be collisions. Considering that particle 2 has a surface charge density $\sigma_{f}$ in external electric field, and $\sigma_{f}$ is estimated due to the polarization under the external electric-field $E$ [10]:

$$
\sigma_{f}=\alpha \cdot \varepsilon_{0} \cdot E \cos \theta
$$

where

$$
\alpha=\frac{3\left(\varepsilon-\varepsilon_{0}\right)}{\varepsilon+2 \varepsilon_{0}} .
$$

Here $\varepsilon_{0}$ represents the vacuum dielectric constant. The dielectric constant of ice is defined as $\varepsilon$, which equals $3 \varepsilon_{0}$. Therefore $\alpha=1.6$.

We assume the initial electric field heads downward vertically. When the initial electric field heads upward, the discussion is similar.

If the relaxation time of collision between particles 1 and
2 lasts as long as $10^{-1}-10^{-2} \mathrm{~s}$, a small amount of positive charge would transfer from particle 2 to particle 1 . The larger ice crystals are typically several hundred microns in diameter. Since the vertical convection can be so energetic that the larger particle can grow into several millimeters in diameter. Here we define $r_{1}(500 \mu \mathrm{m})$ as the radius of particle 1 , and $r_{2}(5 \mathrm{~mm})$ as the radius of particle 2. And Saturn has a small gravity comparative to that on earth $(g=8.96$ $\mathrm{m} / \mathrm{s}^{2}$ at equator) [11].

The positive charge transferred during one collision is estimated as

$$
\Delta q=\pi \cdot r_{1}^{2} \cdot \sigma_{f}=\pi \cdot r_{1}^{2} \cdot \alpha \cdot \varepsilon_{0} \cdot E \cos \theta .
$$

In a period of time, the averaged charge transferred due to the collision between a smaller particle and a larger particle is estimated as

$$
\langle\Delta q\rangle=\pi \cdot r_{1}^{2} \cdot \alpha \cdot \varepsilon_{0} \cdot E \int_{0}^{\frac{\pi}{2}} \cos \theta \sin \theta \mathrm{d} \theta=\frac{\pi}{2} \cdot r_{1}^{2} \cdot \alpha \cdot \varepsilon_{0} \cdot E .
$$

However, we must introduce a factor $\eta$ as an effective collision factor, because the charge is polarized by the exterior electric-field and the polarization charge could not be transferred as easily as free charge, so the collisions between particles of different sizes can not always cause the charge transference. As a result, the charge transferred due to the collisions in a period of time is

$$
\left\langle\Delta q^{\prime}\right\rangle=\langle\Delta q\rangle \cdot \eta=\frac{\pi}{2} \cdot r_{1}^{2} \cdot \alpha \cdot \varepsilon_{0} \cdot E \cdot \eta .
$$

The factor of effective collision $\eta$ determines the charge transferred between particles, which should be very small. Further estimation of the factor of effective collision came from the simulation results. We have simulated the process under different factors of effective collision and determined the factor of effective collision $\eta$ to be $2 \times 10^{-5}$.

Similarly, other factors like the factor of momentum transference $\gamma$, the vertical length of collision region $L$, the area of collision region $S$, the area of the discharge channel $\Delta S$ are also estimated according to the simulation results.

A strong electric field can be built up as long as there is sufficient water abundance [8]. So let us assume that the smaller particles coming from the lower level have a particle density of $n_{1}=1.5 \times 10^{5} \mathrm{~m}^{-3}$ and the larger particles with a density of $n_{2}=10^{4} \mathrm{~m}^{-3}$. The convection process considered at a depth of nearly 8-10 bar (around $200 \mathrm{~km}$ from the tops of the clouds) is only a small part of the moist convection driven by powerful internal thermal heat. Therefore we can suppose that driven force caused by the temperature gradient is persistent during the convective process. As a consequence, the smaller particles will keep the velocity $v$ consistently soon after the collision with larger particles due to the powerful driven force.

However, we have to estimate whether the particles could still be driven by the upward convection or stay still 
in the equilibrium between the driven force and gravity even under the great external electric-field after the charge separation process (the breakdown field could be as large as an order of $10^{7} \mathrm{~V} / \mathrm{m}$ in the Saturn's atmosphere [8]). Let us consider the collision between particles 1 and 2 . The charge accumulated on particle 2 can be estimated as

$$
\left\langle\Delta q^{\prime}\right\rangle_{2}=\frac{\pi}{2} \cdot r_{1}^{2} \cdot \alpha \cdot \varepsilon_{0} \cdot E \cdot \eta \approx 1.11 \times 10^{-15} \mathrm{C} .
$$

Considering that the density of particle 1 is likely 15 fold higher than that of particle 2, the charge accumulated on particle 1 is

$$
\left\langle\Delta q^{\prime}\right\rangle_{1}=\left\langle\Delta q^{\prime}\right\rangle_{2} / 15=7.42 \times 10^{-17} \mathrm{C} .
$$

Here we use the largest electric field $\left(10^{7} \mathrm{~V} / \mathrm{m}\right)$ to do the estimation to make sure that the charge transferred under this strong electric-field will not influence the particles' movement due to the gravity or the up-driven convection.

Therefore the electric field force for particles 1 and 2 can be estimated respectively:

$$
\begin{gathered}
F_{C 1}=\left\langle q^{\prime}\right\rangle_{1} \cdot E \approx 7.42 \times 10^{-10} \mathrm{~N}, \\
F_{C 2}=\left\langle q^{\prime}\right\rangle_{2} \cdot E \approx 1.11 \times 10^{-8} \mathrm{~N} .
\end{gathered}
$$

On the other hand, the gravities of particles 1 and 2 in the atmosphere are

$$
\begin{gathered}
F_{G 1}=\frac{4}{3} \pi \cdot r_{1}^{3} \cdot \rho \cdot g \approx 4.22 \times 10^{-6} N \gg F_{C 1}, \\
F_{G 2}=\frac{4}{3} \pi \cdot r_{2}^{3} \cdot \rho \cdot g \approx 4.22 \times 10^{-3} N \gg F_{C 2} .
\end{gathered}
$$

Here we define $\rho=900 \mathrm{~kg} / \mathrm{m}^{3}$ as the density of ice particles.

Consequently, we can see that the charged particles will not be influenced by the electric field force even under the strongest electric field after the charge separation process. The amount of charge transferred between different particles is so small that the particles would act just as they were after the charge separation and transference.

On the other hand, we should consider the momentum transference caused by the collision between smaller particle and larger particle as well.

If the collision is elastic, we can get the energy conservation equation and the momentum conservation equation in the conference frame of the center of two particles:

$$
\begin{aligned}
\frac{1}{2} m(v \cos \theta)^{2} & =\frac{1}{2} M v_{2}^{2}+\frac{1}{2} m v_{1}^{2}, \\
m v \cos \theta & =M v_{2}+m v_{1} .
\end{aligned}
$$

According to eqs. (12) and (13), we obtain $v_{2}$ as the velocity of the particle 2 after the collision:

$$
v_{2}=\frac{2 m}{m+M} v \cos \theta .
$$

So the momentum transferred on the vertical direction after a collision of a certain angle is

$$
p(\theta)=M v_{2} \cos \theta=\frac{2 m M}{m+M} v \cos ^{2} \theta .
$$

In a short period of time, the averaged momentum transferred due to the collision between a smaller particle and a larger particle can be written as

$$
\langle p(\theta)\rangle=\frac{2 m M}{m+M} v \int_{0}^{\frac{\pi}{2}} \cos ^{2} \theta \sin \theta \mathrm{d} \theta=\frac{2 m M}{3(m+M)} \cdot v .
$$

Since the collision between the particles could be nonelastic in nature state, in some particular conditions, the collision would even causes the particles to adhere to each other or break into pieces if the comparative velocity is too high. Then we introduce a factor $\gamma$ to represent this effect.

$$
\langle P\rangle=\langle p(\theta)\rangle \cdot \gamma=\frac{2 m M}{3(m+M)} \cdot v \cdot \gamma .
$$

Then we need to estimate the momentum transference caused by collision between particles 1 and 2 . The collision between a larger particle and many smaller particles can be described by the momentum conservation:

$$
\begin{aligned}
& n_{1} \pi\left(r_{1}+r_{2}\right)^{2}\left(v-v^{\prime}\right)\langle P\rangle \\
= & n_{1} \pi\left(r_{1}+r_{2}\right)^{2}\left(v-v^{\prime}\right) \frac{2 m M}{3(m+M)} \cdot v \cdot \gamma \\
= & M \frac{\mathrm{d} v^{\prime}}{\mathrm{d} t},
\end{aligned}
$$

where $v^{\prime}$ is the velocity of particle 2 after collisions with many small particles.

Integrating eq. (18), we obtain the relative velocity between the larger particle and the smaller particle:

$$
v-v^{\prime}=v \cdot \mathrm{e}^{-\frac{2 m n_{1} \pi\left(r_{1}+r_{2}\right)^{2} v}{3(M+m)} \cdot \gamma \cdot t} .
$$

The collision frequency $f$ of two kinds of particles with the relative velocity in the collision region (with the volume $V)$ can be estimated as

$$
f=\pi\left(r_{1}+r_{2}\right)^{2} \cdot\left(v-v^{\prime}\right) \cdot n_{1} \cdot n_{2} \cdot V .
$$

According to eqs. (5), (19) and (20), we obtain the charge production rate:

$$
\begin{aligned}
\frac{\mathrm{d} Q}{\mathrm{~d} t} & =\pi\left(r_{1}+r_{2}\right)^{2}\left(v-v^{\prime}\right) n_{1} n_{2} V\left\langle\Delta q^{\prime}\right\rangle \\
& =\frac{\pi^{2}}{2}\left(r_{1}+r_{2}\right)^{2} r_{1}^{2}\left(v-v^{\prime}\right) n_{1} n_{2} \alpha V \varepsilon_{0} E \eta .
\end{aligned}
$$

Based on Ampere Theorem, the relationship between the electric field $E$ and the charge transferred is

$$
\frac{\mathrm{d} \boldsymbol{E}}{\mathrm{d} t}=-\frac{1}{\varepsilon_{0}} \boldsymbol{J}=-\frac{1}{\varepsilon_{0}} \cdot \frac{\boldsymbol{I}}{S^{\prime}} .
$$


We define $S^{\prime}$ to be the horizontal area of collision region, $L\left(5 \times 10^{3}\right)$ the vertical length of collision region.

According to eqs. (21) and (22), we obtain

$$
\begin{aligned}
\frac{\mathrm{d} E}{\mathrm{~d} t} & =\frac{1}{\varepsilon_{0}} \frac{\mathrm{d} Q}{\mathrm{~d} t \cdot S^{\prime}} \\
& =\frac{\pi^{2}}{2} \cdot\left(r_{1}+r_{2}\right)^{2} \cdot r_{1}^{2} \cdot\left(v-v^{\prime}\right) \cdot n_{1} \cdot n_{2} \cdot \alpha \cdot E \cdot \eta \cdot L \\
& =\frac{\pi^{2}}{2}\left(r_{1}+r_{2}\right)^{2} \cdot r_{1}^{2} \cdot n_{1} \cdot n_{2} \cdot \alpha \cdot E \cdot \eta \cdot L \cdot v \cdot \mathrm{e}^{-\frac{2 m n_{1} \pi\left(r_{1}+r_{2}\right)^{2} v}{3(M+m)} \cdot \gamma \cdot t} .
\end{aligned}
$$

Integrating eq. (23), we get the electric-field $E$ in the atmosphere:

$$
E=E_{0} \cdot \exp \left[\frac{3(m+M) \pi \cdot r_{1}^{2} \cdot n_{2} \cdot \alpha \cdot \eta \cdot L}{4 m \cdot \gamma}\left(1-\mathrm{e}^{-\frac{2 m n_{1} \pi\left(r_{1}+r_{2}\right)^{2} v}{3(M+m)} \cdot \gamma \cdot t}\right)\right] .
$$

This electric field is shown in Figure 2.

From Figure 2, we can see that the time spend on the process of charge separation to reach the ultimate electric field is variable due to the variation of the velocity of vertical convection.

\section{Estimation of the breakdown electric field and the discharge process}

The atmospheric electric breakdown field is determined by the background gas and the local air pressure on Saturn. The structure of Saturn's atmosphere is illustrated following the equilibrium cloud condensation model. The particle density in Saturn's hydrogen and helium atmosphere at standard conditions $\left(1 \mathrm{bar}\right.$ and $\left.0^{\circ} \mathrm{C}\right)$ is of the order of $10^{25} \mathrm{~m}^{-3}$ [8]. However, the lightning originates from a level with pressure

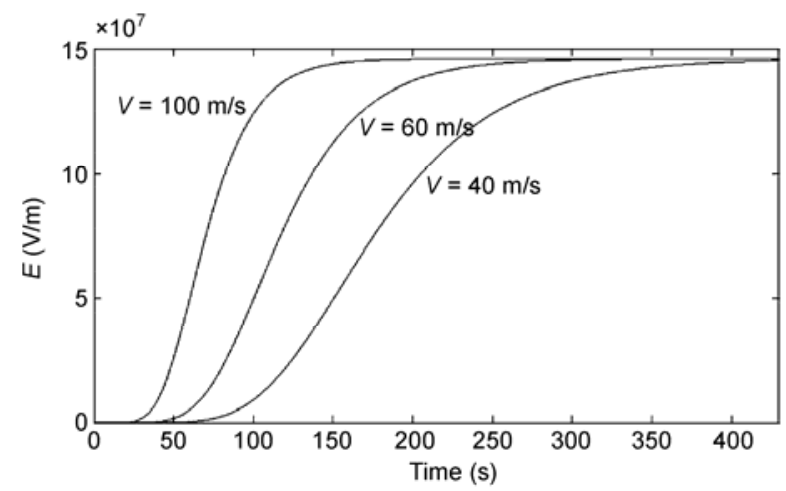

Figure 2 The external electric field's growth as the function of time. Here we define the factor of momentum transference $\gamma$ to be $5 \times 10^{-2}, E_{0}$ to be $1 \mathrm{~V} / \mathrm{m}$, and the vertical length of collision region $L$ to be $5 \times 10^{3} \mathrm{~m}$. The label $V=100 \mathrm{~m} / \mathrm{s}$ refers to the velocity of the upward moist convection of the smaller particles. of $8-10$ bar and temperature of around $275 \mathrm{~K}$. According to

$$
p V=n R T,
$$

we can estimate the local gas particle density as order of $10^{26} \mathrm{~m}^{-3}$.

Then we can obtain the breakdown electric field in such condition. The free length of an electron is estimated as

$$
\bar{\lambda}=\frac{1}{\sqrt{2} n \pi d^{2}} \approx 2.25 \times 10^{-7} \mathrm{~m},
$$

where we define $d$ as the diameter of hydrogen or helium molecules, and $d$ equals to $10^{-10} \mathrm{~m}$.

The kinetic energy of an electron got from the external electric field within one free path of electron is

$$
\frac{1}{2} m_{e} v_{e}^{2}=E \cdot e \cdot \bar{\lambda}
$$

If this kinetic energy is greater than the energy of ionization:

$$
\frac{1}{2} m_{e} v_{e}^{2} \geqslant W_{i}
$$

where $W_{i}$ is the ionization energy of the hydrogen or helium molecules (the helium's ionization energy is much higher than that of hydrogen, so we estimate $W_{i}$, the ionization energy of hydrogen to be of order of $5 \mathrm{eV}$ ), the atom would be ionized. As a result, two electrons are produced. Similarly, these electrons will cause more molecules to be ionized; this process is what we called avalanche ionization.

Therefore the breakdown electric field can be estimated as

$$
E=2.22 \times 10^{7} \mathrm{~V} / \mathrm{m} \text {, }
$$

which is of the same order with the breakdown electric field observed: $E=1.5 \times 10^{7} \mathrm{~V} / \mathrm{m} \quad$ [8].

When such external electric field reaches the high level of $10^{7} \mathrm{~V} / \mathrm{m}$, then avalanche ionization begins. The density of ionized particle especially the electrons can be written as

$$
n=n_{0} \cdot(\chi \cdot 2)^{\frac{x}{\bar{\lambda}}}
$$

where $n_{0}$ is the initial density of electrons, $x$ is the travelling distance of the avalanche electric current, and $\chi$ is the factor of ionization. The collision of the accelerated electrons would not always cause the target atom to be ionized.

As we can see, the electron density would increase exponentially due to the avalanche ionization, and soon a limit will reach, because the gas particle density is limited to $10^{26}$ $\mathrm{m}^{-3}$. Let us assume that the gas particle could be ionized completely, which means that the electron density would soon increase to an order of $10^{26} \mathrm{~m}^{-3}$, and then the local gas would be transformed to plasma completely in a very narrow channel. This electric conductance is 


$$
\sigma=\frac{n e^{2} \bar{\lambda}}{2 m_{e} \bar{v}} \approx 6.3 \times 10^{5} \mathrm{~A} \cdot \mathrm{m}^{-1} \cdot \mathrm{V}^{-1} .
$$

And the electric current density is obtained:

$$
j=\sigma \cdot E .
$$

After the formation of the plasma discharge channel, the accumulated charge due to the moist convection will all travel through this particular channel because the electric conductance here is extremely high. We can establish a model to demonstrate the discharge procedure.

The electric field between the charged cloud layers can be estimated as

$$
E=\frac{\sigma_{e}}{\varepsilon_{0}}=\frac{Q}{S \cdot \varepsilon_{0}}
$$

where $S$ is the area of the clouds and $Q$ is the charge accumulated in the cloud.

According to the eqs. (30)-(32), we obtain the conductive current:

$$
I=\Delta S \cdot j=\Delta S \cdot \sigma \cdot E=\frac{\sigma \cdot Q}{S \cdot \varepsilon_{0}} \cdot \Delta S=-\frac{\mathrm{d} Q}{\mathrm{~d} t},
$$

where $\Delta S$ is the area of the discharge channel.

Integrating the eq. (33), we get the quantity of remnant charge:

$$
Q=Q_{0} \exp \left\{-\frac{\sigma \cdot \Delta S}{S \cdot \varepsilon_{0}} \cdot t\right\} .
$$

If we assume $Q_{0}$ as the initial amount of charge caused by the convection process $\left(Q_{0}\right.$ is of the order of several hundred coulomb), and do the evaluation of these parameters: $S$ to be several square kilometers, and $\Delta S$ to be an order of $10^{-3} \mathrm{~m}^{2}$, then remnant charge is shown in Figure 3 .

From Figure 3 we can see that the declination of the quantity of charge is extremely rapid. Several hundred

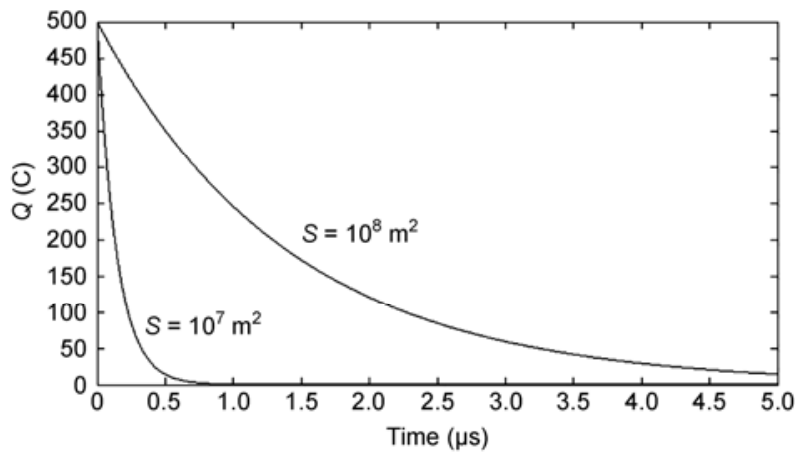

Figure 3 The decay of the amount of charge accumulated in the vertical convection. coulomb of charge would decay within several microseconds. The accurate decaying time is depended on the area of the discharge channel, the area of the charged cloud and the amount of charge in the charged cloud region.

\section{Conclusion and discussion}

We established two models to interpret the charge separation process and the discharge process respectively. Generally speaking, the charge separation process is due to the powerful moist convection caused by the internal thermal heat. The lightning could be very energetic because of the large breakdown field in the Saturn's atmosphere, so the powerful convection will cause the electric field to the critical point of $10^{7} \mathrm{~V} / \mathrm{m}$. Then the energy an electron can get from the acceleration in the electric field is high enough to cause the ionization of another electron from its atom. Thus the avalanche ionization is triggered. The energy accumulated during the thermal-induced convection is released within a very short time. This discharge process that we called lightning can be very powerful because of the energetic convection which cause the charge generation or separation and the great critical breakdown field in Saturn's atmosphere.

This work was supported by the Knowledge Innovation Program of the Chinese Academy of Sciences (KZZD-EW-01-4, KZCX2-EW-QN501) and National Natural Science Foundation of China (41074118, 40890163, 41121003).

1 Yang J, Feng G L. A gigantic jet event observed over a thunderstorm in mainland China. Chin Sci Bull, 2012, 57: 4791-4800

2 Wang J X. Solar activity studies: From a magnetohydrodynamics description to a plasma perspective. Chin Sci Bull, 2012, 57: 13621368

3 Ning Z J. Power conversion factor in solar flares. Chin Sci Bull, 2012, 57: 1397-1404

4 Zong Q G, Wang Y F, Yuan C J, et al. Fast acceleration of "killer" electrons and energetic ions by interplanetary shock stimulated ULF waves in the inner magnetosphere. Chin Sci Bull, 2011, 56: 11881201

5 Wu D J, Feng H Q. Basic plasma processes in solar-terrestrial activities. Chin Sci Bull, 2012, 57: 1353-1356

6 Fischer G, Kurth W S, Dyudina U A, et al. Analysis of a giant lightning storm on Saturn. Icarus, 2007, 190: 528-544

7 Dyudina U A, Andrew P I, Ewald S P, et al. Lightning storms on Saturn observed by Cassini ISS and RPWS during 2004-2006. Icarus, 2007, 190: 545-555

8 Fischer G, Gurnett D A, Kurth W S, et al. Atmospheric electricity at Saturn. Space Sci Rev, 2008, 137: 271-285

9 Dyudina U A, Ingersoll A P, Ewald S P, et al. Detection of visible lightning on Saturn. Geophys Res Lett, 2010, 37: L09205

10 Jackson J D. Classical Electrodynamics. 3rd ed. New York: John Wiley \& Sons, 2004. 159

11 Hueso R, Sanchez-Lavega A. A three-dimensional model of moist convection for the giant planets II: Saturn's water and ammonia moist convective storms. Icarus, 2004, 172: 255-271

Open Access This article is distributed under the terms of the Creative Commons Attribution License which permits any use, distribution, and reproduction in any medium, provided the original author(s) and source are credited. 